\title{
Quantitative Analysis of Epinephrine in Human Plasma Samples Using Kinetic Fluorometric Method Combined with Second-order Calibration
}

\author{
Shu-Fang LI, Hai-Long Wu ${ }^{\dagger}$ A-Lin XIA, Shao-Hua ZHU, Jin-Fang NIE, Yong-Jie YU, and \\ Ru-Qin YU
}

State Key Laboratory of Chemo/Biosensing and Chemometrics, College of Chemistry and Chemical Engineering, Hunan University, Changsha 410082, P. R. China

\begin{abstract}
A kinetic fluorometric method was proposed for the quantitative determination of epinephrine (EP) in human plasma samples with the aid of second-order calibration methods based on alternating trilinear decomposition (ATLD) and alternating fitting residue (AFR) algorithms. It was based on that EP could be gradually converted to a highly fluorescent intermediate product by an oxidation reaction, and further to a non-fluorescent degradation product (o-quinone). These methodologies fully exploit the second-order advantage of the employed three-way kinetic fluorescence data, allowing the concentrations of EP to be quantified even in the presence of uncalibrated interferences. The average recoveries obtained from ATLD and AFR with a factor number of $2(N=2)$ were $100.7 \pm 3.3$ and $100.4 \pm 2.2 \%$, respectively. In addition, elliptical joint confidence region (EJCR) tests as well as figures of merit (FOM) were employed to evaluate the accuracy of the two algorithms.
\end{abstract}

(Received November 12, 2008; Accepted May 18, 2009; Published October 10, 2009)

Epinephrine (1-(3,4-dihydroxyphenyl)-2-methylaminoethanol), (EP), is one of the naturally occurring catecholamine hormones secreted by the adrenal medulla together with sympathetic nerve terminals, and acts as a neurotransmitter in the mammalian central nervous system. Pharmaceutically, it is now widely employed in the treatment of heart failure and cardiac surgery, but some adverse reactions to EP have also been reported. ${ }^{1}$ Furthermore, changes in the EP level have been correlated with stress, heart disease and other health problems. Thus, it is essential to accomplish quantitative determinations of EP in biological fluids, such as urine, plasma and serum.

A variety of analytical methods have been utilized for the determination of EP and other catecholamines in various biological fluids and pharmaceutical preparations. ${ }^{2-14}$ These mainly include high-performance liquid chromatography (HPLC) with electrochemical detection ${ }^{2}$ or mass spectrometry, ${ }^{3}$ flow injection analysis (FIA) techniques, ${ }^{4}$ electrochemical detection with various modified electrodes, ${ }^{5,6}$ capillary electrophoresis $\quad(\mathrm{CE}){ }^{7} \quad$ chemiluminescence $\quad(\mathrm{CL}),{ }^{8}$ spectrophotometry, ${ }^{9,10}$ and fluorescence detection. ${ }^{11-14}$ However, most of these methods usually involve careful pretreatment steps when analyzing biological matrices. Furthermore, the selective extraction of catecholamines from a biological sample requires complex washing procedures, which are not selective enough, and introduction variability in the recovery steps. Apparently, methodologies requiring no tedious pretreatment steps would have advantages in diagnostic time, cost, labor, and having no analytes lost during the recovery steps and being more suitable for routine laboratories.

Several spectroscopic methods for the determination of EP

† To whom correspondence should be addressed.

E-mail: hlwu@hnu.cn usually involve its oxidation reaction, which has been widely studied. ${ }^{9-12}$ Some studies ${ }^{13,14}$ have also described the application of kinetic methods based on the oxidation reaction in quantitative analysis of catecholamines or qualitative analysis of their kinetic behavior. It has been proved that spectrofluorometric methods have a capacity for providing high sensitivity, and the fluorescence spectrum information can be conveniently recorded in a single, easily available instrument. For the aforementioned reasons, spectrofluorometric methods are suitable for the determination of EP. However, it is still difficult to be accomplished for the quantification of EP in biological samples when traditional fluorometric methodologies are used without any previous separation procedure, due to the presence of a potentially interfering biological background. In these cases, we could resort to the application of chemometric methods, which might light a new avenue to simple experimentation utilizing "mathematical separation" to replace the "physical or chemical separation" of uncalibrated background or interferences by the chemometric separation of their signals..$^{15,16}$

In recent years, with the increasing popularity of modern hyphenated instruments capable of generating a multidimensional data array for each sample, multiway data arrays have been prominently useful for the quantitative analysis of complex samples. The attractive merit derived from three-way data arrays is attributed to that the decomposition of a three-way data array built with response matrices measured for many samples is often unique, allowing relative concentrations and spectral profiles of individual sample components to be extracted directly. That is, the analysis of several components of interest can be quantified even in the presence of unknown interferences, a property usually called the "second-order advantage". ${ }^{17,18}$ Thus far, several second-order calibration methods have been developed for a three-way data array to solve complex chemical 
problems, such as the parallel factor analysis (PARAFAC), ${ }^{19,20}$ the alternating trilinear decomposition (ATLD), ${ }^{15}$ the self-weighed alternating trilinear decomposition (SWATLD), ${ }^{21}$ and the alternating fitting residue (AFR) algorithms. ${ }^{22}$

Kinetic studies could provided both quantitative and qualitative information with the objective of describing the underlying reaction model with kinetic parameters, and are attractive to use in conjunction with chemometrics. There are many successful applications employing this methodology, which has been an appealing domain in analytical chemistry. For example, Tan et al. studied a kinetic system that involved simultaneous degradations of chlorophyll $a$ and $b$ using a three-way resolution method based on PARAFAC analysis. ${ }^{23}$ Moreover, an interesting application of kinetic determination coupled with chemometrics was paid special attention concerning drug analysis ${ }^{24}$ in biological samples and contaminants in environmental monitoring. 25,26

In the present work, a kinetic fluorometric method for the quantitative analysis of EP in human plasma samples was demonstrated, which depends on the transformation of EP to the corresponding fluorescent intermediate product and further to $o$-quinone. We employed three-way kinetic fluorescence data combined with second-order calibration methods based on the ATLD and AFR algorithms. The emission spectra profiles as well as the kinetic evolution profiles of the intermediate and concentration information were resolved and studied for this complex system without any time-consuming pretreatment. Besides solving a specific problem, the present paper represents a contribution to the recognition and application of a kinetic method in combination with chemometrics. In addition, the figures of merit of both ATLD and AFR algorithms, such as the sensitivity (SEN), selectivity (SEL) and limit of detection (LOD), were estimated for the proposed methods, and the accuracy of both algorithms was also studied by using the elliptical joint confidence region (EJCR) test. These results further prove that both of the algorithms can give accurate results, respectively, in spite of the fact that the performance of AFR is somewhat better than that of ATLD.

\section{Theory}

\section{Reaction kinetics}

EP could be converted to a highly fluorescent intermediate product (trihydroxyindole derivative) by oxidation under an alkaline condition, and further to a non-fluorescent degradation product (o-quinone). The principle for the oxidation reaction of EP is described in Fig. 1.

Here, we use potassium hexacyanoferrate(III) as an oxidizer, with zinc sulfate as a catalyst in the presence of boric acid buffer (pH 9.06) and ascorbic acid as an antioxidant. All reactants are added in accord with an appropriate adding step and in excess, which means that the concentration of the intermediate product is rate determining in this reaction. The reaction mechanism has been discussed previously. Wu et al. ${ }^{14}$ have proposed that this reaction followed pseudo-first order kinetics and reported the rate expression. The following equation indicates the according theoretical expression:

$$
[\mathrm{B}]_{\mathrm{t}}=\frac{k_{1}[\mathrm{EP}]_{0}}{k_{2}-k_{1}}\left(e^{-\mathrm{k}_{1} \mathrm{t}}-e^{-\mathrm{k}_{2} \mathrm{t}}\right),
$$

where $[\mathrm{B}]_{\mathrm{t}}$ denotes the concentration of the intermediate at time $t$, and $[\mathrm{EP}]_{0}$ is the starting concentration of EP, $k_{1}$ and $k_{2}$ are the two rate constants in the reaction. Hence, it is expected that the fluorescence intensity of the intermediate is linearly related to

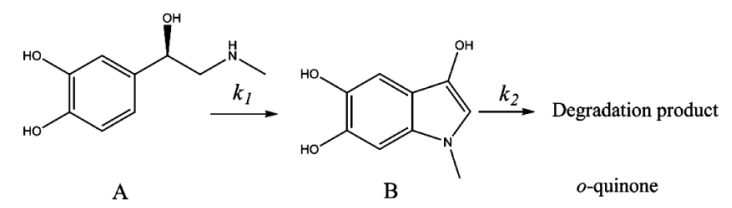

Fig. 1 Simplified reaction scheme for EP. EP (A) was oxidized to a trihydroindole derivative (B) and further to degradation product (o-quinone).

the EP over time. The intermediate is the only compound whose fluorescence intensity varied with time under an emission range at $\lambda_{\mathrm{em}} 439-550 \mathrm{~nm}$ when the excitation wavelength was fixed at $390 \mathrm{~nm}$.

\section{Trilinear model}

In the case of a kinetic-emission fluorescence determination, a three-way data array $\underline{\mathbf{X}}$ can be produced by stacking a series of emission-time fluorescence spectra obtained for each of the $K$ samples consisting of calibration samples and prediction samples. Appropriate dimensions of such an array are $I \times J \times K$ ( $I$ is the number of emission wavelengths and $J$ is the number of time points). A trilinear component model for such a three-way array $\underline{\mathbf{X}}$ has the following form:

$$
\begin{aligned}
& x_{\mathrm{ijk}}=\sum_{\mathrm{n}=1}^{\mathrm{N}} a_{\mathrm{in}} b_{\mathrm{jn}} c_{\mathrm{kn}}+e_{\mathrm{ijk}} \\
& (i=1,2, \ldots, I ; j=1,2, \ldots, J ; k=1,2, \ldots, K),
\end{aligned}
$$

where $x_{\mathrm{ijk}}$ is the fluorescent intensity of sample $k$ at the emission wavelength $i$ and time point $j$, and $N$ denotes the number of factors, which should be considered as the total number of fluorescing species, consisting of the components of interest and the background as well as un-calibrated interferences. $a_{\text {in }}$ is the element $(i, n)$ of an $I \times N$ matrix $\mathbf{A}$ with relative emission profiles of the $N$ species; $b_{\mathrm{jn}}$ is the element $(j, n)$ of a $J \times N$ matrix $\mathbf{B}$ with relative time profiles of the $N$ species; $c_{\mathrm{kn}}$ is the element $(k, n)$ of a $K \times N$ matrix $\mathbf{C}$ with relative concentrations of the $N$ species in $K$ samples; and $e_{\mathrm{ijk}}$ represents the element of an $I \times J \times K$ three-way residual array, $\underline{\mathbf{E}}$.

An important characteristic of $\underline{\mathbf{X}}$ is that it can be uniquely decomposed, which can provide access to emission spectral profiles (A) and corresponding time profiles (B) as well as the relative concentrations $(\mathbf{C})$ of individual components in the $\mathrm{K}$ samples, even in the presence of chemically unknown interferences. The property fabricates the basis of the second-order advantage.

\section{$A T L D$ and $A F R$}

In the present study, the ATLD and AFR algorithms were implemented to resolve the three-way data array consist of the kinetic fluorescence spectra of the intermediate and the natural interferences, mainly originating from the complicated plasma background.

An alternating trilinear decomposition (ATLD) was proposed by $\mathrm{Wu}$ et al. ${ }^{15}$ which is an improvement on the traditional PARAFAC algorithm without any constraints. It is based on the alternating least-squares principle, and utilizes an iterative procedure involving a Moore-Penrose generalized inverse with singular value decomposition (SVD). The loss function to be minimized is the sum of squares of the residual matrices, which may be written as: 


$$
\begin{aligned}
& \sigma_{1}(\mathbf{A})=\sum_{\mathrm{i}=1}^{\mathrm{I}}\left\|\mathbf{X}_{\mathrm{i..}}-\mathbf{B} \operatorname{diag}\left(\mathbf{a}_{(\mathrm{i})}\right) \mathbf{C}^{\mathrm{T}}\right\|_{\mathrm{F}}^{2}, \\
& \sigma_{2}(\mathbf{B})=\sum_{\mathrm{j}=1}^{\mathrm{J}}\left\|\mathbf{X}_{\mathrm{j} .}-\mathbf{C} \operatorname{diag}\left(\mathbf{b}_{(\mathrm{j})}\right) \mathbf{A}^{\mathrm{T}}\right\|_{\mathrm{F}}^{2}, \\
& \sigma_{3}(\mathbf{C})=\sum_{\mathrm{k}=1}^{\mathrm{K}}\left\|\mathbf{X}_{. . \mathrm{k}}-\mathbf{A} \operatorname{diag}\left(\mathbf{c}_{(\mathrm{k})}\right) \mathbf{B}^{\mathrm{T}}\right\|_{\mathrm{F}}^{2},
\end{aligned}
$$

where $\|\bullet\|_{\mathrm{F}}^{2}$ denotes the Frobenius matrix norm. Equations (3) (5) can be considered to be equivalent to each other owing to the cyclic symmetry the property of trilinear model. The ATLD algorithm minimizes alternately one of the above-mentioned loss functions, and then one can obtain the relative emission matrix $\mathbf{A}$, relative time evolution matrix $\mathbf{B}$ and relative concentrations matrix C. More detailed information about this algorithm is available in Ref. 15.

An alternating fitting residue (AFR) algorithm ${ }^{22}$ uses the least squares-based AF error to construct three objective functions, and then decomposes the array $\underline{\mathbf{X}}$ by alternately minimizing the above-mentioned three objective functions (AF error). In the original literature, an exhaustive content of the algorithm was described.

According to our practical experience, when the chosen model dimensionality is much more than the actual number of components, generally, a model fitting error and a deviation between the predicted and actual results will arise. That is to say, the proposed algorithms can perform well with more acceptable resolution and calibration when the most appropriate factor has been extracted. With the above consideration, the present work estimates the number of factors using the core consistency diagnostic (CORCONDIA), which is computed for a number of trial components, and $N$ is set as one less the number for which the core consistency value drops from 100 to less than $50 .{ }^{27}$

\section{Figures of merit}

Figures of merit (FOM), such as sensitivity (SEN), selectivity (SEL) and limit of detection (LOD) are frequently used for method comparison. In second-order calibration, the idea of FOM is easy to be comprehended by resorting to the useful concept of net analyte signal (NAS), ${ }^{28}$ which is defined as the part of the signal that relates uniquely to the analyte of interest. More details regarding the determination of NAS can be found in the critical review by Faber et al. ${ }^{31}$ Different approaches for the calculation of the FOM have been extensively discussed in the literature. ${ }^{29-32}$

The sensitivity is estimated as the NAS at unit concentration, and the selectivity is the ratio between the sensitivity and the total signal. The following equations can be obtained to estimate the SEN and SEL in this presently studied case:

$$
\begin{aligned}
& \mathrm{SEN}=k\left\{\left[\left(\mathbf{A}^{\mathrm{T}} \mathbf{A}\right) *\left(\mathbf{B}^{\mathrm{T}} \mathbf{B}\right)\right]^{-1}\right\}_{\mathrm{nn}}^{-1 / 2}, \\
& \mathrm{SEL}=\left\{\left[\left(\mathbf{A}^{\mathrm{T}} \mathbf{A}\right) *\left(\mathbf{B}^{\mathrm{T}} \mathbf{B}\right)\right]^{-1}\right\}_{\mathrm{nn}}^{-1 / 2},
\end{aligned}
$$

where $n n$ designates the $(n, n)$ element of a matrix and $k$ is the total signal for component $n$ at unit concentration, which is also a parameter for converting scores to concentrations; the symbol * denotes the Hadamard product. Note that when the second-order advantage is employed, Eqs. (6) and (7) imply that SEN and SEL are sample-specific and cannot be defined for the multivariate method as a whole. In such cases, average values for a set of samples can be estimated and reported.

The limit of detection (LOD) ${ }^{33-35}$ can be calculated as follows:

$$
\operatorname{LOD}=3.3 s(0)
$$

where $s(0)$ is the standard deviation in the predicted concentration estimated for three different background blank plasma samples, in the ATLD and AFR algorithms.

\section{Experimental}

\section{Reagents and solutions}

EP was obtained from the National Institute for the Control of Pharmaceutical and Biological Products (Changsha, China). The human plasma was purchased from the Blood Center in Changsha. All other reagents were of analytical-reagent grade. Doubly distilled water was used throughout the experiments. A $47.6 \mu \mathrm{g} \mathrm{ml}^{-1}$ stock solution was prepared by dissolving EP in $5 \mathrm{mM}$ hydrochloric acid solution and remained in the dark at $4^{\circ} \mathrm{C}$ for several days. Fresh working solutions were prepared daily from the stock solution by diluting with doubly distilled water. A $0.2 \mathrm{M}$ boric buffer solution was prepared from $\mathrm{H}_{2} \mathrm{BO}_{3}$ and $\mathrm{NaB}_{4} \mathrm{O}_{7} \cdot 10 \mathrm{H}_{2} \mathrm{O}$ at $\mathrm{pH} 9.06$. Aqueous solution of $3.51 \mathrm{mM}$ $\mathrm{K}_{3} \mathrm{Fe}(\mathrm{CN})_{6}, 3.64 \mathrm{mM} \mathrm{ZnSO}_{4}$ and $10 \mathrm{mM}$ ascorbic acid were prepared each day.

\section{Apparatus}

Measurements of the fluorescence spectra signals were performed on an F-4500 fluorescence spectrophotometer (Hitachi, Japan), which was equipped with a $150-\mathrm{W}$ continuous xenon lamp and interfaced to a personal computer. A $1.00 \mathrm{~cm}$ quartz cell was used at room temperature in all cases.

\section{Software}

In the MATLAB environment all home-made programs were written and further used for data analysis. All calculations were carried out on a microcomputer under the Windows XP operating system.

\section{Analytical methodology}

Prior to analysis, the linear analytical range was investigated for EP. It was found that by linear regression between different EP concentrations and the relative fluorescence intensity of corresponding intermediate product. The linear range obtained was $0.0852-1.3632 \mu \mathrm{g} \mathrm{ml}^{-1}$.

The first five samples, containing EP concentrations of 0.1904, $0.3808, \quad 0.5712, \quad 0.7616, \quad 0.9520 \mu \mathrm{g} \mathrm{ml}^{-1}$, were used as concentration calibration samples. Sample Nos. 6 - 11 contained both EP and human plasma diluted with water $(\mathrm{v}: \mathrm{v}=1: 5)$. Both the calibration and human plasma samples were prepared according to the following steps, and the final analyte concentrations in human plasma samples should be within the calibration concentration range. The spectra of the reagent blank (without EP and plasma) were recorded in triplicate during the whole analysis procedure to subtract the influence of blank solutions. Three blank drug-free plasma samples (with plasma and without EP) were also determined in order to estimate $s(0)$, and then to calculate the limit of detection. The fluorescence spectra of the solution were measured as quickly as possible after the preparation was finished. For each sample, the reagent adding steps were in accordance with the following order of priority: 1) Appropriate amounts of EP working solution were placed in $10.00 \mathrm{ml}$ brown volumetric flasks, and $2 \mathrm{ml}$ human plasma would like to join in the human plasma samples. 

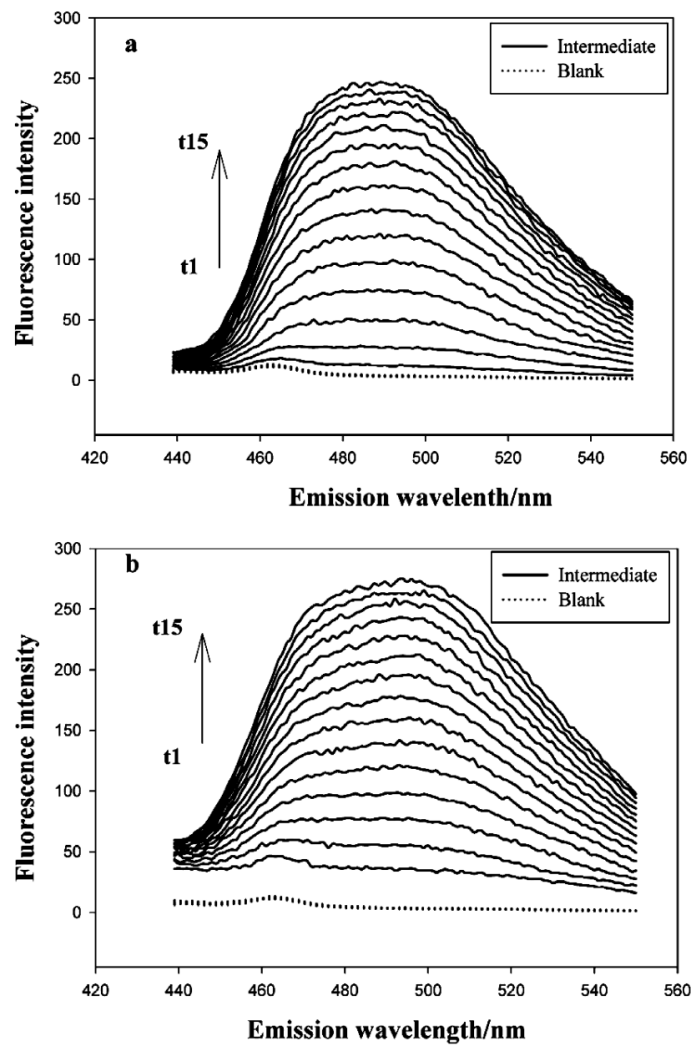

Fig. 2 Two-dimensional kinetic plots of sample 2 (a) and sample 7 (b) at 15 consecutive time points in the emission range $439-550 \mathrm{~nm}$ when a constant $390 \mathrm{~nm}$ excitation wavelength was fixed.

A $1.0-\mathrm{ml} \mathrm{ZnSO}_{4}$ solution was employed. 2) Timed the process with a watch when $1 \mathrm{ml} \mathrm{K}_{3} \mathrm{Fe}(\mathrm{CN})_{6}$ solution was added. 3) After $30 \mathrm{~s}$, added $1 \mathrm{ml}$ boric buffer solution of $\mathrm{pH} 9.06$. 4) After $30 \mathrm{~s}$, put $1 \mathrm{ml}$ of an ascorbic acid solution in, diluted to the mark with water and mixed thoroughly. Fluorescence spectra were recorded starting after $120 \mathrm{~s}$ from step 2 .

\section{Measurement}

An F-4500 fluorescence spectrophotometer was set in the wavelength scan mode, the cycle time was $2 \mathrm{~min}$, replicates were 15 and the detection wavelength was chosen in an emission range of $\lambda_{\mathrm{em}} 439-550 \mathrm{~nm}$ with an interval of $1 \mathrm{~nm}$ when the excitation wavelength was fixed at $390 \mathrm{~nm}$. The emission and excitation slit widths were both $5 \mathrm{~nm}$, and the scanning rate of the instrument was maintained at $1200 \mathrm{~nm} \mathrm{~min}^{-1}$. The kinetic curve was recorded and saved for data processing. The fluorescence spectra were exported in the ASCII format from the instrument software to MATLAB. Under the above-mentioned chemical conditions, a two-dimensional data array of size $112 \times$ 15 (emission wavelengths $\times$ time points) was obtained for each sample. The combination of data matrices from 11 samples constituted a three-way data array of size $112 \times 15 \times 11$ (emission wavelengths $\times$ time points $\times$ sample numbers).

\section{Results and Discussion}

\section{Emission spectra of intermediate}

As can be seen in Fig. 2, two-dimensional kinetic plots of sample 2 (Fig. 2a) and sample 7 (Fig. 2b) were revealed, respectively. Sample 2 contained only EP and sample 7 contained both EP and plasma. The fluorescence spectra for

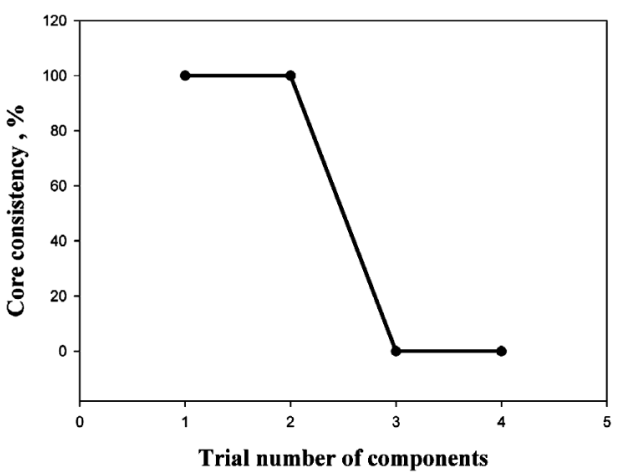

Fig. 3 Core consistency values as a function of the trial number of components in the analysis of human plasma samples.

intermediate product of EP (solid lines) as well as blank solution (dotted lines) along with time points were displayed when the excitation wavelength was fixed at $390 \mathrm{~nm}$ in the emission wavelength range $\left(\lambda_{\mathrm{em}}: 439-550 \mathrm{~nm}\right)$. It was observed that the fluorescence signals of sample 7 were enhanced by the plasma, and had the same trend as sample 2. Note that the fluorescence intensity of the intermediate increased along with time, and those of the blank almost had no variation. The above-mentioned measurement parameters were selected for all of the samples throughout experiments. A two-dimensional data array $(112 \times$ 15) was obtained for each sample by stacking the sequential fluorescent intensities along the time coordinate, and then two-dimensional data of calibration sets and human plasma samples were collected. Consequently, we could obtain a three-way data array $(112 \times 15 \times 11)$ that consisted of the fluorescent intensities as a function of the emission wavelength, time points, and concentration of samples.

\section{Core consistency diagnostic (CORCONDIA)}

Prior to using the trilinear decomposition algorithms, the factor numbers of the mixture had to be estimated. Core consistency diagnostic (CORCONDIA) is a very useful tool for this intention. All data sets were utilized for the core consistency evaluation, using one to four factors. While the core consistency values are negative, they are caused to equal zeros. An appropriate number of components is considered to be obtained when the core consistency drops from a high value (usually above $60 \%$ ) to a low value. For this test, the result is shown in Fig. 3. The analysis using CORCONDIA based on the ATLD or AFR algorithms indicates that two factors are necessary for plasma samples, because the utilization of more factors leads to a great decrease in the core consistency. A possible explication for this result is that the chemical species in plasma samples is very similar, and ATLD and AFR did not resolve these into separated factors, but considered one factor, ${ }^{36}$ i.e. one is corresponding to the intermediate and the other to the plasma background.

\section{Determination of EP in human plasma sample}

Second-order calibration requires the decomposition of a three-way data array and the regression of the relative concentration of the components of interest in the sample space against the corresponding standard concentrations. For the decomposition of a three-way data array in the present study, the ATLD and AFR algorithms were implemented to resolve the kinetic fluorescence spectra of intermediate and natural interferences, mainly originating from the complicated plasma 

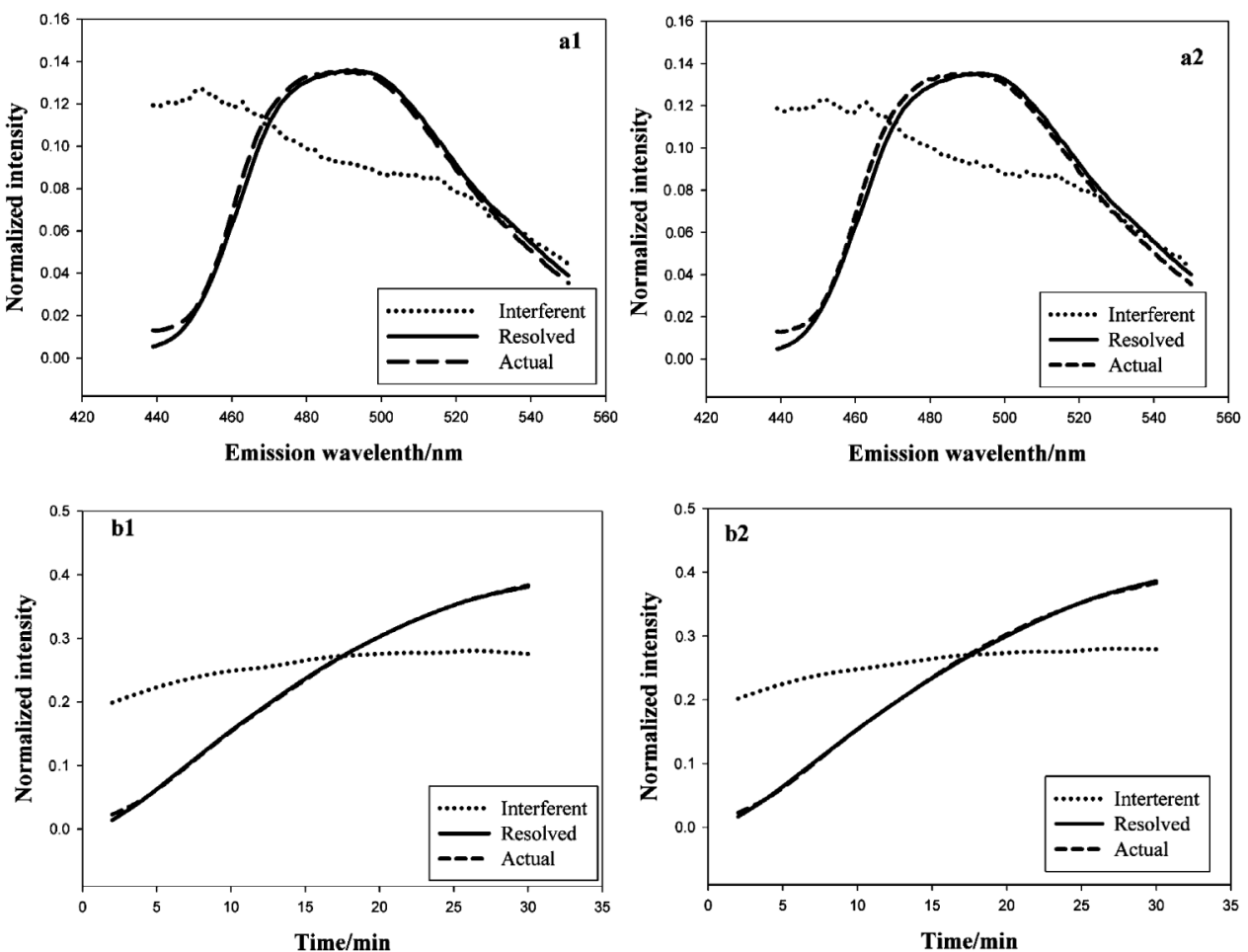

Fig. 4 Resolved emission (a1, a2) and the corresponding time (b1, b2) profiles using the proposed algorithms on the three-way data set when component number was chosen as two $(N=2)$ : (a1), (b1) ATLD; (a2), (b2) AFR.

Table 1 Resolved concentrations of prediction samples and figures of merit (FOM) of EP in plasma using ATLD and AFR

\begin{tabular}{|c|c|c|c|c|c|c|c|c|c|c|}
\hline & \multicolumn{6}{|c|}{ Sample } & \multicolumn{4}{|c|}{ Figures of merit } \\
\hline & 6 & 7 & 8 & 9 & 10 & 11 & $\begin{array}{l}\text { RMSEP/ } \\
\mu \mathrm{g} \mathrm{ml}^{-1}\end{array}$ & $\begin{array}{c}\text { (SEN/AFU)/ } \\
\mathrm{ml} \mu \mathrm{g}^{-1}\end{array}$ & SEL & $\begin{array}{c}\mathrm{LOD} / \\
\mu \mathrm{g} \mathrm{ml}^{-1}\end{array}$ \\
\hline $\mathrm{Real} / \mu \mathrm{g} \mathrm{ml}^{-1}$ & 0.2856 & 0.3808 & 0.4760 & 0.5712 & 0.6664 & 0.7616 & & & & \\
\hline $\mathrm{ATLD} / \mu \mathrm{g} \mathrm{ml}^{-1}$ & 0.2716 & 0.3773 & 0.4789 & 0.6018 & 0.7063 & 0.7474 & 0.0222 & $7.276 \times 10^{3}$ & 0.6061 & 0.0030 \\
\hline $\mathrm{AFR} / \mu \mathrm{g} \mathrm{ml}^{-1}$ & 0.2782 & 0.3804 & 0.4813 & 0.5998 & 0.6894 & 0.7402 & 0.0154 & $7.394 \times 10^{3}$ & 0.5986 & 0.0028 \\
\hline
\end{tabular}

background, and to directly estimate the concentration of the interesting component, which may furthest minimize the sample pretreatment.

The emission spectra profiles as well as the kinetic evolution profiles of the intermediate and concentration information were resolved from the decomposition of the kinetic emission fluorescence data array obtained for both the calibration and predicted samples by using the ATLD (Figs. 4a1 and b1) and AFR (Figs. 4a2 and b2) algorithms with a factor number of two $(N=2)$. Figure 4 shows the resolved emission spectral profiles and the corresponding time profiles in plasma samples together with the actual ones. The loadings associated with the emission mode were shown in Fig. 4a, and the loadings associated with the time mode were shown in Fig. 4b. These emission and time evolution spectral profiles were collected into matrices $\mathbf{A}$ and $\mathbf{B}$, respectively. In Fig. 4, the solid lines represent the loadings of the intermediate; the dotted lines represent the loadings for the inherent interferences, mainly derivied from human plasma. Besides, the dashed lines denote the actual spectral profiles. It can be appreciated that the resolved intermediate spectral profiles are quite similar to the actual ones, which confirms the accuracy and reliability of the proposed strategy, and satisfactory predicted results of the EP concentrations maybe expected.

The prediction results for the human plasma samples using both the ATLD and AFR algorithms with $N=2$ are summarized in Table 1. For EP, the average predicted recoveries gained from ATLD and AFR are 100.7 \pm 3.3 and 100.4 $\pm 2.2 \%$, respectively. The correlation coefficients of ATLD and AFR are 0.9982 and 0.9995 . The results of the root-mean-square error of prediction (RMSEP) obtained from the two algorithms are $0.2222 \mu \mathrm{g} \mathrm{ml}^{-1}$ for ATLD and $0.0154 \mu \mathrm{g} \mathrm{ml}^{-1}$ for AFR. These results indicate that both second-order calibration algorithms provide for the analysis with a satisfactory prediction capacity to quantitative EP in human plasma samples.

The study based on second-order calibration also furnished analytical figures of merit. Table 1 collects the figures of merit including SEN, SEL as well as LOD for the methods based on ATLD and AFR. It seems that the overall prediction abilities of both algorithms are satisfactory. There is no significant difference in the precision and accuracy between the two algorithms, although slightly favoring the AFR algorithm. In order to acquire further insight into the accuracy of the proposed algorithms, a linear-regression analysis of actual versus prediction concentration was applied. ${ }^{37}$ The estimated intercept 


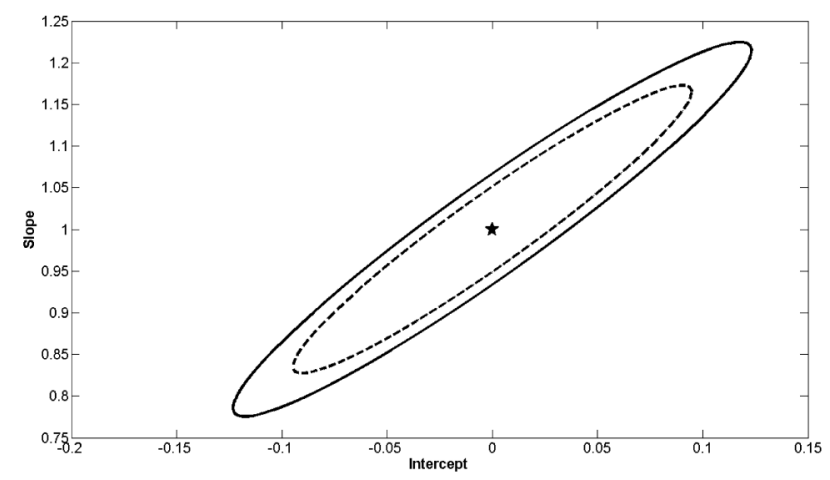

Fig. 5 EJCRs for both ATLD (solid line) and AFR (dashed line) The pentacle indicates the ideal points $(0,1)$.

and slope were compared with their ideal values of 0 and 1 , based on an elliptical joint confidence region (EJCR) test. ${ }^{38}$ Figure 5 gives the results of EJCRs for both the ATLD and AFR calibration methods. It shows that the ideal point $(0,1)$, labeled with a pentacle $(\star)$, lies in both the EJCRs and the elliptic size corresponding to the AFR algorithm is smaller than that related to the ATLD algorithm. These factors further prove that both of the algorithms can give accurate results, respectively, although the performance of AFR is somewhat better than that of ATLD.

\section{Conclusions}

The kinetic fluorescence data for EP can be successfully decomposed by using the ATLD and AFR algorithms, thanks to the second-order advantage. The results obtained in this work indicate that the kinetic fluorometric method combined with second-order calibration methods based on the trilinear decomposition algorithms is an attractive alternative strategy for the quantification of EP in biological samples. Several improvements of the method are possible, and it is expected that the potential advantages of this determination, such as rapidity and low cost, can be even more highlighted by considering the possibility of automating the proposed method. The application in our work is only a limited example of the enormous potentiality of this strategy in the biomedical analytical fields. More applications in other fields, such as food, environmental and agronomical analysis can also be imagined.

\section{Acknowledgements}

The authors wish to express their deep appreciation to the National Science Foundation of China (Grant Nos. 20775205, 20435010) and the National Basic Research Program (No. 2007CB216404) for financial supports while completing this work.

\section{References}

1. H. Yao, Y. Y. Sun, X. H. Lin, Y. H. Tang, A. L. Liu, G. W. Li, W. Li, and S. B. Zhang, Anal. Sci., 2007, 23, 677.

2. B. A. Patel, M. Arundell, K. H. Parker, M. S. Yeoman, and D. O'Hare, J. Chromatogr., B, 2005, 818, 269.

3. A. Törnkvist, P. J. R. Sjöberg, K. E. Markides, and J. Bergquist, J. Chromatogr., B, 2004, 801, 323.

4. L. K. Abdulrahman, A. M. Al-Abachi, and M. H. Al-Qaissy, Anal. Chim. Acta, 2005, 538, 331.
5. M. Zhou, L. P. Guo, Y. Hou, and X. J. Peng, Electrochim. Acta, 2008, 53, 4176.

6. K. Hayashi, Y. Iwasaki, R. Kurita, K. Sunagawa, O. Niwa, and A. Tate, J. Electroanal. Chem., 2005, 579, 215.

7. M. M. Hsieh and H. T. Chang, J. Chromatogr., A, 2006, 1102, 302.

8. M. Tsunoda and K. Imai, Anal. Chim. Acta, 2005, 541, 13.

9. X. G. Wu, R. X. Cai, L. Zhang, and M. Xiao, Anal. Chim. Acta, 2001, 448, 257.

10. X. G. Wu and R. X. Cai, Anal. Chim. Acta, 2002, 464, 153.

11. P. Cañizares and M. D. L. de Castro, Anal. Chim. Acta, 1995, 317, 335.

12. R. H. Christenson and C. D. McGlothlin, Anal. Chem., 1982, 54, 2015.

13. R. P. H. Nikolajsen, K. S. Booksh, A. M. Hansen, and R. Bro, Anal. Chim. Acta, 2003, 475, 137.

14. X. G. Wu, R. X. Cai, Z. X. Lin, and J. K. Cheng, Anal. Chim. Acta, 1996, 325, 105.

15. H. L. Wu, M. Shibukawa, and K. Oguma, J. Chemom., 1998, 12,1 .

16. J. F. Nie, H. L. Wu, A. L. Xia, S. H. Zhu, Y. C. Bian, S. F. Li, and R. Q. Yu, Anal. Sci., 2007, 23, 1377.

17. B. R. Kowalski and M. B. Seasholtz, J. Chemom., 1991, 5, 129.

18. K. S. Booksh and B. R. Kowalski, Anal. Chem., 1994, 66, 782A.

19. R. A. Harshman, UCLA Working Papers in Phonetics, 1970, 16,1 .

20. R. Bro, Chemom. Intell. Lab. Syst., 1997, 38, 149.

21. Z. P. Chen, H. L. Wu, J. H. Jiang, Y. Li, and R. Q. Yu, Chemom. Intell. Lab. Syst., 2000, 52, 75.

22. A. L. Xia, H. L. Wu, D. M. Fang, Y. J. Ding, L. Q. Hu, and R. Q. Yu, Anal. Sci., 2006, 22, 1189.

23. Y. X. Tan, J. H. Jiang, H. L. Wu, H. Cui, and R. Q. Yu, Anal. Chim. Acta, 2000, 412, 195.

24. A. García-Reiriz, P. C. Damiani, and A. C. Olivieri, Talanta, 2007, 71, 806.

25. S. H. Zhu, H. L. Wu, A. L. Xia, Q. J. Han, Y. Zhang, and R. Q. Yu, Talanta, 2008, 74, 1579.

26. M. V. Bosco, M. P. Callao, and M. S. Larrechi, Anal. Chim. Acta, 2006, 576, 184.

27. R. Bro, "Muti-way Analysis in the Food Industry, Models, Algorithms and Applications", Doctoral Thesis, 1998 University of Amsterdam.

28. A. Lorber, Anal. Chem., 1986, 58, 1167.

29. N. J. Messick, J. H. Kalivas, and P. M. Lang, Anal. Chem., 1996, 68, 1572.

30. Y. Wang, O. S. Borgen, B. R. Kowalski, M. Gui, and F. Turecek, J. Chemom., 1993, 7, 117.

31. K. Faber, A. Lorber, and B. R. Kowalski, J. Chemom., 1997, 11, 95.

32. A. C. Olivieri, Anal. Chem., 2005, 77, 4936.

33. A. Lorber, K. Faber and B. R. Kowalski, Anal. Chem., 1997, 69, 1620.

34. R. Boqué, J. Ferré, and N. M. Faber, F. X. Rius, Anal. Chim. Acta, 2002, 451, 313.

35. A. C. Olivieri and N. M. Faber, Chemom. Intell. Lab. Syst., 2004, 70, 75 .

36. M. G. Trevisan and R. J. Poppi, Anal. Chim. Acta, 2003, 493, 69.

37. J. A. Arancibia and G. M. Escandar, Talanta, 2003, 60, 1113.

38. A. G. González, M. A. Herrador, and A. G. Asuero, Talanta, 1999, 48, 729 . 